\title{
The New Semianalytical Technique for the Solution of Fractional- Order Navier-Stokes Equation
}

\author{
Nehad Ali Shah $\left(\mathbb{D},{ }^{1}\right.$ Mounirah Areshi $\mathbb{D}^{2},{ }^{2}$ Jae Dong Chung, ${ }^{1}$ and Kamsing Nonlaopon $\mathbb{D}^{3}$ \\ ${ }^{1}$ Department of Mechanical Engineering, Sejong University, Seoul 05006, Republic of Korea \\ ${ }^{2}$ Mathematics Department, College of Science, University of Tabuk, Tabuk, Saudi Arabia \\ ${ }^{3}$ Department of Mathematics, Faculty of Science, Khon Kaen University, Khon Kaen 40002, Thailand
}

Correspondence should be addressed to Kamsing Nonlaopon; nkamsi@kku.ac.th

Received 1 March 2021; Revised 29 March 2021; Accepted 3 April 2021; Published 20 April 2021

Academic Editor: Qifeng Zhang

Copyright ( 2021 Nehad Ali Shah et al. This is an open access article distributed under the Creative Commons Attribution License, which permits unrestricted use, distribution, and reproduction in any medium, provided the original work is properly cited.

In this paper, we introduce a modified method which is constructed by mixing the residual power series method and the Elzaki transformation. Precisely, we provide the details of implementing the suggested technique to investigate the fractional-order nonlinear models. Second, we test the efficiency and the validity of the technique on the fractional-order Navier-Stokes models. Then, we apply this new method to analyze the fractional-order nonlinear system of Navier-Stokes models. Finally, we provide 3-D graphical plots to support the impact of the fractional derivative acting on the behavior of the obtained profile solutions to the suggested models.

\section{Introduction}

The fractional-order Navier-Stokes equation (NSE) has been extensively analyzed. These equations model the fluid motion defined by several physical processes, such as the movement of blood, the ocean's current, the flow of liquid in vessels, and the airflow around an aircraft's arms [1-3]. The classical NSEs were generalized by El-Shahed and Salem [4] by replacing the first time derivative with a Caputo fractional derivative of order $\alpha$, where $0<\alpha \leq 1$. Using Hankel transform, Fourier sine transform, and Laplace transform, the researchers achieved the exact solution for three different equations. In 2006, Momani and Odibat [5] solve fractionalorder NSEs using the Adomian decomposition method. Ganji et al. [6] applied an analytical technique, the homotopy perturbation method, for solving the fractional-order NSEs in polar coordinates, and the results achieved were expressed in a closed form. Singh and Kumar [7] solved the fractionalorder reduced differential transformation method (FRDTM) to achieve an approximated analytical result of fractionalorder multidimensional NSE. Oliveira and Oliveira [8] analyzed the residual power series method (RPSM) to find the result of the nonlinear fractional-order two-dimensional
NSEs. Zhang and Wang [9] suggested numerical analysis for a class of NSEs with fractional-order derivatives; Ravindran, the exact boundary controllability of Galerkin approximations of a Navier-Stokes system for soret convection [10]; and Cibik and Yilmaz, the Brezzi-Pitkaranta stabilization and a priori error analysis for the Stokes control [11].

Some researchers mix two powerful techniques to achieve another result technique to solve equations and systems of fractional-order NSEs. Below, we define some of these combinations: a combination of the Laplace transformation and Adomian decomposition method; Kumar et al. [12] introduced the homotopy perturbation transform method (HPTM), combined Laplace transformation with the homotopy perturbation method, and solved fractionalorder NSEs in a tube. Jena and Chakraverty [13] implemented the homotopy perturbation transformation method (HPETM), and this technique consists in the mixture of Elzaki transformation technique and homotopy perturbation technique; Prakash et. al [1] suggested $q$-homotopy analysis transformation technique to achieve a result of coupled fractional-order NSEs. This technique mixture of the Laplace transformation and residual power series method is defined: 


$$
\left\{\begin{array}{l}
D_{\tau}^{\alpha} u+(u \cdot \nabla) u=-\frac{1}{\rho} \nabla p+v \nabla^{2} u, \quad 0<\alpha \leq 1, \\
\nabla u=0
\end{array}\right.
$$

where $D_{\tau}^{\alpha} u$ is the Caputo derivative of order $\alpha, u$ is the velocity vector, $\tau$ is the time, $v$ is kinematics viscosity, $p$ is the pressure, and $\rho$ is the density.

In this work, we consider two special cases. First, we consider unsteady, one-dimensional motion of a viscous fluid in a tube. The fractional-order Navier-Stokes equations in cylindrical coordinates that governs the flow field in the tube are given by

$$
D_{\tau}^{\alpha} u+P+v\left(\frac{\partial^{2} u}{\partial \psi^{2}}+\frac{1}{\psi} \frac{\partial u}{\partial \psi}\right), \quad 0<\alpha \leq 1,
$$

with initial condition

$$
u(\psi, 0)=g(\psi)
$$

where $P=-1 / \rho \partial p / \partial z$ and $g(\psi)$ is a function depending only on $\psi$.

Consider that the fractional-order two-dimensional Navier-Stokes equations is defined as

$$
\begin{aligned}
& D_{\tau}^{\alpha} u=\rho_{0}\left(\frac{\partial^{2}}{\partial \psi^{2}} u+\frac{\partial^{2}}{\partial \varphi^{2}} u\right)-u \frac{\partial}{\partial \psi} u-v \frac{\partial}{\partial \varphi} u+g \\
& D_{\tau}^{\alpha} v=\rho_{0}\left(\frac{\partial^{2}}{\partial \psi^{2}} v+\frac{\partial^{2}}{\partial \varphi^{2}} v\right)-u \frac{\partial}{\partial \psi} v-v \frac{\partial}{\partial \varphi} v-g
\end{aligned}
$$

with initial conditions

$$
\begin{aligned}
& u(\psi, \varphi, \tau)=f(\psi, \varphi), \\
& v(\psi, \varphi, \tau)=g(\psi, \varphi),
\end{aligned}
$$

where $u=u(\psi, \varphi, \tau), v=v(\psi, \varphi, \tau), \rho, \tau, p$ denote as constant density, time, and pressure, respectively. $\psi, \varphi$ are the spatial components, and $f(\psi, \varphi)$ and $g(\psi, \varphi)$ are two functions depending only on $\psi$ and $\varphi$.

The residual power series method (RPSM) is a simple and efficient technique for constructing a power series result for extremely linear and nonlinear equations without perturbation, linearization, and discretization. Unlike the classical power series technique, the RPS approach does not need to compare the coefficients of the corresponding terms and a recursion relation is not required. This approach calculates the power series coefficients by a series of algebraic equations of one or more variables, and its reliance on derivation, which is much simpler and more precise than integration, which is the basis of most other solution approaches, is the main advantage of this methodology. This method is, in effect, an alternative strategy for obtaining theoretical results for the fractional-order partial differential equations [14].

The RPSM was introduced as an essential tool for assessing the power series solution's values for the first and second-order fuzzy DEs [15]. It has been successfully implemented in the approximate result of the generalized LaneEmden equation [16], which is a highly nonlinear singular $\mathrm{DE}$, in the inaccurate work of higher-order regular DEs [17], in the solution of composite and noncomposite fractional-order DEs [18], in predicting and showing the diversity of results to the fractional-order boundary value equations [19], and in the numerical development of the nonlinear fractional-order $\mathrm{KdV}$ and Burgers equation [20], in addition to some other implementations [21-23], and recently, it has been applied to investigate the approximate result of a fractional-order two-component evolutionary scheme [24].

This paper introduces the modified analytical technique: the residual power series transform method (RPSTM) is implemented to investigate the fractional-order NS equations. The result of certain illustrative cases is discussed to explain the feasibility of the suggested method. The results of fractional-order models and integral-order models are defined by using the current techniques. The new approach has lower computing costs and higher rate convergence. The suggested method is also constructive for addressing other fractional orders of linear and nonlinear PDEs.

\section{Preliminaries}

Definition 1. The Abel-Riemann of fractional operator $D^{\alpha}$ of order $\alpha$ is given as [25-27]

$D^{\alpha} v(\zeta)= \begin{cases}\frac{d^{j}}{d \zeta^{j}} v(\zeta), & \alpha=j, \\ \frac{1}{\Gamma(j-\alpha)} \frac{d}{d \zeta^{j}} \int_{0}^{\zeta} \frac{v(\zeta)}{(\zeta-\psi)^{\alpha-j+1}} d \psi, & j-1<\alpha<j,\end{cases}$

where $j \in \mathbb{Z}^{+}, \alpha \in \mathbb{R}^{+}$and

$$
D^{-\alpha} v(\zeta)=\frac{1}{\Gamma(\alpha)} \int_{0}^{\zeta}(\zeta-\psi)^{\alpha-1} v(\psi) d \psi, \quad 0<\alpha \leq 1 .
$$

Definition 2. The fractional-order Abel-Riemann integration operator $J^{\alpha}$ is defined as [25-27]

$$
J^{\alpha} v(\zeta)=\frac{1}{\Gamma(\alpha)} \int_{0}^{\zeta}(\zeta-\psi)^{\alpha-1} v(\zeta) d \zeta, \quad \zeta>0, \alpha>0
$$

The operator of basic properties

$$
\begin{aligned}
J^{\alpha} \zeta^{j} & =\frac{\Gamma(j+1)}{\Gamma(j+\alpha+1)} \zeta^{j+\psi}, \\
D^{\alpha} \zeta^{j} & =\frac{\Gamma(j+1)}{\Gamma(j-\alpha+1)} \zeta^{j-\psi} .
\end{aligned}
$$

Definition 3. The Caputo fractional operator ${ }^{C} D^{\alpha}$ of $\alpha$ is defined as [25-27] 


$$
{ }^{C} D^{\alpha} v(\zeta)= \begin{cases}\frac{1}{\Gamma(j-\alpha)} \int_{0}^{\zeta} \frac{v^{j}(\psi)}{(\zeta-\psi)^{\alpha-j+1}} d \psi, & j-1<\alpha<j, \\ \frac{d^{j}}{d \zeta^{j}} v(\zeta), & j=\alpha .\end{cases}
$$

Definition 4. The fractional-order Caputo operator of Elzaki transform is given as [25-27]

$E\left[D_{\zeta}^{\alpha} g(\zeta)\right]=s^{-\alpha} E[g(\zeta)]-\sum_{k=0}^{j-1} s^{2-\alpha+k} g^{(k)}(0)$, where $j-1<\alpha<j$.

Definition 5. A power series definition of the form [14]

$$
\sum_{m=0}^{\infty} P_{m}(\tau-\psi)^{m \alpha}=P_{0}+P_{1}(\tau-\psi)+P_{2}(\tau-\psi)^{2 \alpha}+\cdots
$$

where $0 \leq m-1<\alpha \leq m$ and $\tau \leq \psi$ is called fractional power series (FPS) about $\psi$, where $P_{m}$ are the constants called the coefficients of the series. If $\psi=0$, then the fractional power series will be reduced to the fractional Maclaurin series.

Theorem 6. Assume that $f_{0}$ has a fractional power series representation at $\tau=\psi$ of the form [14]

$$
f_{o}(\tau)=\sum_{m=0}^{\infty} P_{m}(\tau-\psi)^{m \alpha} .
$$

For $m \in N U\{0\}$, if $D^{m \alpha} f_{o}(\tau)$ are continuous on $(\psi, \psi+$ $R_{o}$ ), then the coefficients $P_{m}$ can be written as

$$
P_{m}=\frac{D^{m \alpha} f_{o}(\psi)}{\Gamma(1+m \alpha)}
$$

where $\psi \leq \tau<\psi+R_{o}$, and $R_{o}$ is the radius of convergence.

Definition 7. The expansion of power series of the form [14]

$$
\sum_{m=0}^{\infty} G_{m}(\phi)(\tau-\psi)^{m \alpha}
$$

is said to be multifractional power series at $\tau=\psi$, where $G_{m}$ $(\phi)$ are the coefficients of multifractional power series.

Theorem 8. Let us assume that $u_{o}(\phi, \tau)$ which has the multifractional power representation at $\tau=\psi$ can be written as [14]

$$
u_{o}(\phi, \tau)=\sum_{m=0}^{\infty} G_{m}(x)(\tau-\psi)^{m \alpha}
$$

For $m \in N U\{0\}$, if $D_{\tau}^{m \alpha} u_{o}(\phi, \tau)$ are the continuous on $I_{o}$ $\times\left(\psi, \psi+R_{o}\right)$, then the coefficients $G_{m}$ are given by

$$
G_{m}(x)=\frac{D_{\tau}^{m \alpha} u_{o}(\phi, \tau)}{\Gamma(1+m \alpha)}
$$

where $\psi \in I_{o}$ and $\psi \leq \tau<\psi+R_{o}$.

So, we can write the fractional power expansion of $u_{o}(\phi, \tau)$ of the form

$$
\mu(\phi, \tau)=\sum_{m=0}^{\infty} \frac{D_{\tau}^{m \alpha} \mu(\phi, \tau)}{\Gamma(1+m \alpha)}(\tau-\psi)^{m \alpha},
$$

which is the generalized Taylor expansion. If we consider $\alpha=1$, then the generalized Taylor formula will be converted to classical Taylor series.

Corollary 9. Let us assume that $\mu(\phi, \varphi, \tau)$ has a multifractional power series representation about $\tau=\psi$ as [14]

$$
u_{o}(\phi, \varphi, \tau)=\sum_{m=0}^{\infty} G_{m}(\phi, \varphi)(\tau-\psi)^{m \alpha} .
$$

For $m \in N U\{0\}$ if $D_{\tau}^{m \alpha} \mu(\phi, \varphi, \tau)$ are continuous on $I_{1} \times$ $I_{2} \times\left(\psi, \psi+R_{o}\right)$, then

$$
G_{m}(\psi, \varphi)=\frac{D_{\tau}^{m \alpha} \mu(\phi, \varphi, \tau)}{\Gamma(1+m \alpha)},
$$

where $(\phi, \varphi) \in I_{1} \times I_{2}, \psi \leq \tau<\psi+R_{o}$.

\section{The Procedure of RPSTM}

In this section, we explain the steps of RPSTM for solving the fractional-order partial differential equation

$$
D_{\tau}^{\alpha} u(\psi, \tau)=a D_{\psi}^{2} u(\psi, \tau)+b u(\psi, \tau)-c u^{q}(\psi, \tau),
$$

with initial condition

$$
u(\psi, 0)=f_{0}(\psi) .
$$

First, we use the Elzaki transform to (21); we get

$\mathscr{E}\left[D_{\tau}^{\alpha} u(\psi, \tau)\right]=a \mathscr{E}\left[D_{\psi}^{2} u(\psi, \tau)\right]+b \mathscr{E}[u(\psi, \tau)]-c \mathscr{E}\left[u^{q}(\psi, \tau)\right]$

By the fact that $\mathscr{E}\left[D_{\tau}^{\alpha} u(\psi, \tau)\right]=1 / s^{\alpha} \mathscr{E}[u(\psi, \tau)]-s^{1-\alpha} u(x$ $, 0)$ and using the initial condition (22), we can write (23) as

$$
\begin{aligned}
U(\psi, s)= & s^{2} f_{0}(\psi)+s^{\alpha} a D_{x}^{2} U(\psi, s)+b s^{\alpha} U(\psi, s) \\
& -c s^{\alpha} \mathscr{C}^{-1}\left[(\mathscr{E}[U(\psi, s)])^{q}\right],
\end{aligned}
$$

where $U(\psi, s)=\mathscr{E}[u(\psi, \tau)]$.

Second, we define the transform function $U(\psi, s)$ as the following formula:

$$
U(\psi, s)=\sum_{n=0}^{\infty} s^{n \alpha+1} f_{n}(x) .
$$


We write the $k$ th truncated series of (25) as

$$
U(\psi, s)=\sum_{n=0}^{\infty} s^{n \alpha+1} f_{n}(x)=s^{2} f_{0}(x)+\sum_{n=1}^{\infty} s^{n \alpha+1} f_{n}(x) .
$$

As stated in [25], the definition of Elzaki residual function to $(25)$ is

$$
\begin{aligned}
\mathscr{E} \operatorname{Res}(\psi, s)= & U(\psi, s)-f_{0}(x) s^{2}-a s^{\alpha} D_{x}^{2} U(\psi, s)-b s^{\alpha} U(\psi, s) \\
& +c s^{\alpha} \mathscr{E}^{-1}\left[(\mathscr{E}[U(\psi, s)])^{q}\right]
\end{aligned}
$$

and the $k$ th Elzaki residual function of (27) is

$$
\begin{aligned}
\mathscr{E} \operatorname{Res}(\psi, s)= & U_{k}(\psi, s)-f_{0}(x) s^{2}-a s^{\alpha} D_{x}^{2} U_{k}(\psi, s) \\
& -b s^{\alpha} U_{k}(\psi, s)+c s^{\alpha} \mathscr{E}\left[\left(\mathscr{E}^{-1}\left[U_{k}(\psi, s)\right]\right)^{q}\right] .
\end{aligned}
$$

Third, we expand a few of the properties arising in the basic RPSM to find out certain facts:

(i) $\mathscr{E} \operatorname{Res}(\psi, s)=0$ and $\lim _{k \rightarrow \infty} \mathscr{E} \operatorname{Res} s_{k}(\psi, s)=\mathscr{E} \operatorname{Res}(\psi, s)$ for each $s>0$

$$
\lim _{k \rightarrow \infty} \mathscr{E} \operatorname{Res}(\psi, s)=0 \Rightarrow \lim _{k \rightarrow \infty} s \mathscr{E} \operatorname{Re} s_{k}(\psi, s)=0
$$

(ii) $\lim _{k \rightarrow \infty} s^{k \alpha+1} \mathscr{E} \operatorname{Res}(\psi, s)=\lim _{k \rightarrow \infty} s^{k \alpha+1} \mathscr{E} \operatorname{Re} s_{k}(\psi, s)$

$$
=0,0<\alpha \leq 1, k=1,2,3, \cdots
$$

Furthermore, to evaluate the coefficient functions $f_{n}(\psi)$, we can recursively solve the following scheme

$$
\lim _{s \rightarrow \infty}\left(s^{k \alpha+1} \mathscr{E} \operatorname{Re} s_{k}(\psi, s)\right)=0, \quad 0<\alpha \leq 1, k=1,2,3, \cdots .
$$

Finally, we implemented the Elzaki inverse to $U_{k}(\psi, s)$ to achieve the $k$ th approximate supportive solution $u_{k}(\psi, \tau)$

\section{Numerical Results}

Example 1. Consider the time-fractional-order onedimensional NS equation of the form

$$
D_{\tau}^{\alpha} u(\psi, \tau)=P+\frac{\partial^{2} u}{\partial \psi^{2}}+\frac{1}{\psi} \frac{\partial u}{\partial \psi}, \quad 0<\alpha \leq 1 .
$$

Subject to the initial condition

$$
u(\psi, 0)=1-\psi^{2}
$$

Applying Elzaki transform to (31) and using the initial condition given in (32), we get

$$
\begin{aligned}
U(\psi, s)= & s^{2}\left(1-\psi^{2}\right)+s^{\alpha} \mathscr{E}_{\tau}[P]+s^{\alpha} \mathscr{E}_{\tau} \\
& \cdot\left[\mathscr{E}_{\tau}^{-1}\left\{\frac{\partial^{2}}{\partial \psi^{2}} U(\psi, s)\right\}\right]+s^{\alpha} \mathscr{E}_{\tau}\left[\mathscr{E}_{\tau}^{-1}\left\{\frac{1}{\psi} \frac{\partial}{\partial \psi} U(\psi, s)\right\}\right] .
\end{aligned}
$$

The $k$ th truncated term series of (33) is

$$
U_{k}(\psi, s)=s^{2}\left(1-\psi^{2}\right)+\sum_{n=1}^{k} s^{n \alpha+2} f_{n}(\psi)
$$

and the $k$ th Elzaki residual function is

$$
\begin{aligned}
\mathscr{E}_{\tau} \operatorname{Re} s_{k}= & U_{k}(\psi, s)-s^{2}\left(1-\psi^{2}\right)-s^{\alpha+2} P-s^{\alpha} \mathscr{E}_{\tau} \\
& \cdot\left[\mathscr{E}_{\tau}^{-1}\left\{\frac{\partial^{2}}{\partial \psi^{2}} U_{k}(\psi, s)\right\}\right]-s^{\alpha} \mathscr{E}_{\tau}\left[\mathscr{E}_{\tau}^{-1}\left\{\frac{1}{\psi} \frac{\partial}{\partial x} U_{k}(\psi, s)\right\}\right] .
\end{aligned}
$$

Now, to determine $f_{k}(\psi), k=1,2,3, \cdots$, we substitute the $k$ th truncated series (34) into the $k$ th Elzaki residual function (35), multiply the resulting equation by $s^{k \alpha+2}$, and then solve recursively the relation $\lim _{s \rightarrow \infty}\left[s^{k \alpha+2} \operatorname{Re} s_{k}(\psi, s)\right]=0, k=1,2$ $, 3, \cdots$, for $f_{k}(\psi)$. The following are the first several components of the series $f_{k}(\psi, \varphi)$ :

$$
\begin{aligned}
& f_{1}(\psi)=p-4, \\
& f_{2}(\psi)=0, \\
& f_{3}(\psi)=0,
\end{aligned}
$$

Putting the values of $f_{n}(\psi)(n \geq 1)$ in (34), we get

$$
\begin{aligned}
& U(\psi, s)=s^{2}\left(1-\psi^{2}\right)+s^{\alpha+2} f_{1}(\psi)+s^{2 \alpha+2} f_{2}(\psi)+s^{3 \alpha+2} f_{3}(\psi)+\cdots \\
& U(\psi, s)=s^{2}\left(1-\psi^{2}\right)+s^{\alpha+2}(P-4)+s^{2 \alpha+2}(0)+s^{3 \alpha+2}(0)+\cdots
\end{aligned}
$$

$$
U(\psi, s)=s^{2}\left(1-\psi^{2}\right)+s^{\alpha+2}(P-4) .
$$

Using inverse Elzaki transform to (38), we get

$$
u(\psi, \tau)=1-\psi^{2}+\frac{(P-4) \tau^{\alpha}}{\Gamma(\alpha+2)}
$$

Putting $\alpha=1$, we have

$$
u(\psi, \tau)=1-\psi^{2}+(P-4) \tau
$$

In Figure 1, the RPSTM and the exact results of Example 1 at $\alpha=1$ are shown by plots (a) and (b), respectively. From the given figures, it can be seen that both the exact and the EDM results are in close contact with each other. Also, in the Figure 2 subgraph, the RPSTM results of Example 1 are calculated at different fractional-order $\alpha=0.8$ and 0.6 . It is investigated that fractional-order problem results are 

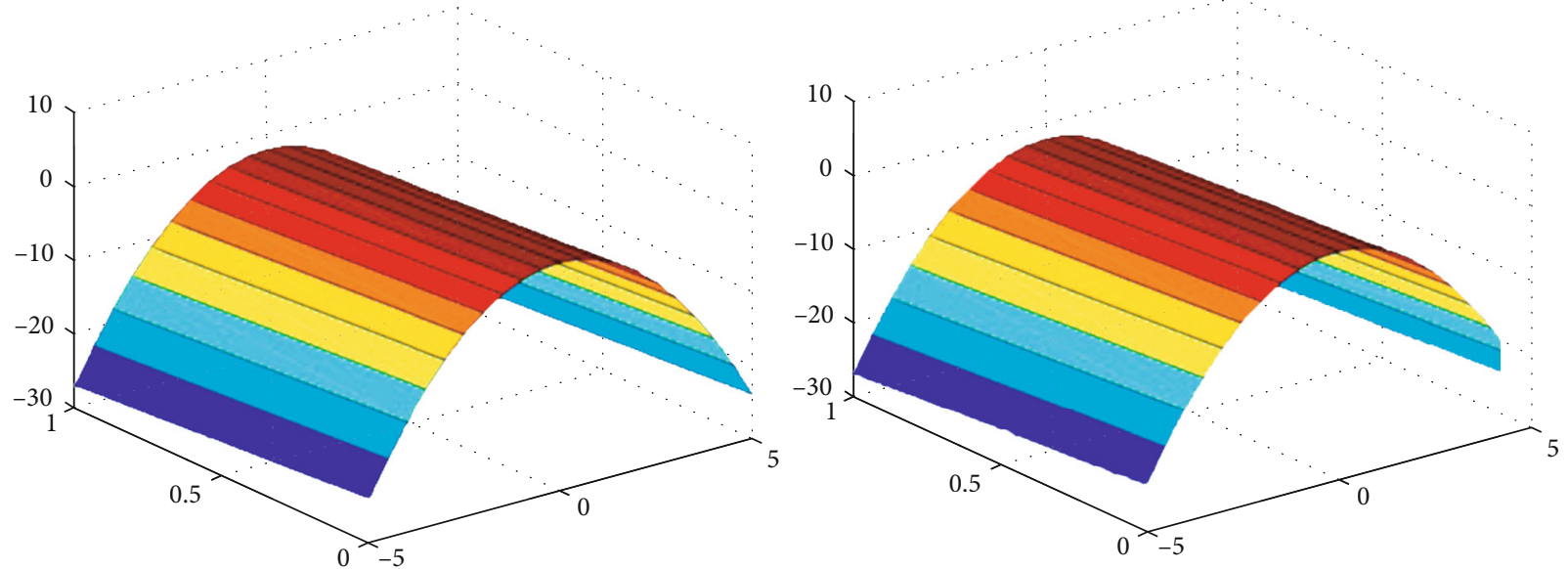

Figure 1: Graph of exact and analytical results of Problem 1.
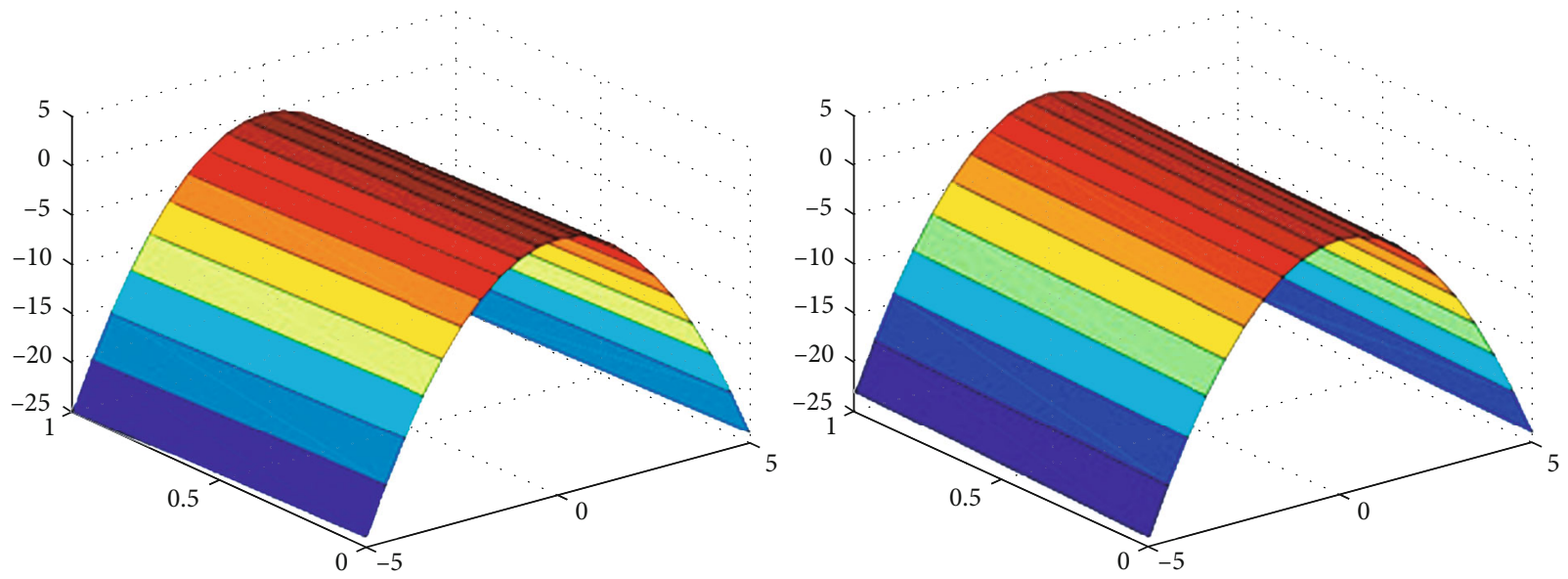

FIgure 2: The fractional order of $\alpha=0.8$ and 0.6 of Problem 1.

convergent to an integer-order effect as fractional-order analysis to integer-order. The same phenomenon of convergence of fractional-order solutions towards integral-order solutions is observed.

Example 2. Consider the fractional-order one-dimensional NS equation of the form

$$
D_{\tau}^{\alpha} u(\psi, \tau)=\frac{\partial^{2} u}{\partial \psi^{2}}+\frac{1}{\psi} \frac{\partial u}{\partial \psi}, \quad 0<\alpha \leq 1
$$

Subject to the initial condition,

$$
u(\psi, 0)=\psi
$$

Applying Elzaki transform to (41) and using the initial condition given in (42), we get

$U(\psi, s)=s^{2}(\psi)+s^{\alpha} \mathscr{E}_{\tau}\left[\mathscr{E}_{\tau}^{-1}\left\{\frac{\partial^{2}}{\partial \psi^{2}} U(\psi, s)\right\}\right]+s^{\alpha} \mathscr{C}_{\tau}\left[\mathscr{E}_{\tau}^{-1}\left\{\frac{1}{\psi} \frac{\partial}{\partial x} U(\psi, s)\right\}\right]$.
The $k$ th truncated term series of (43) is

$$
U_{k}(\psi, s)=s^{2}(\psi)+\sum_{n=1}^{k} s^{n \alpha+2} f_{n}(\psi)
$$

and the $k$ th Elzaki residual function is

$$
\begin{aligned}
\mathscr{E}_{\tau} \operatorname{Re} s_{k}= & U_{k}(\psi, s)-s^{2}(\psi)-s^{\alpha} \mathscr{E}_{\tau}\left[\mathscr{E}_{\tau}^{-1}\left\{\frac{\partial^{2}}{\partial \psi^{2}} U_{k}(\psi, s)\right\}\right] \\
& -s^{\alpha} \mathscr{E}_{\tau}\left[\mathscr{E}_{\tau}^{-1}\left\{\frac{1}{\psi} \frac{\partial}{\partial x} U_{k}(\psi, s)\right\}\right] .
\end{aligned}
$$

Now, to determine $f_{k}(\psi), k=1,2,3, \cdots$, we substitute the $k$ th truncated series (44) into the $k$ th Elzaki residual function (45), multiply the resulting equation by $s^{k \alpha+2}$, and then solve recursively the relation $\lim _{s \rightarrow \infty}\left[s^{k \alpha+2} \operatorname{Re} s_{k}(\psi, s)\right]=0, k=1,2$ $, 3, \cdots$, for $f_{k}(\psi)$. The following are the first several components of the series $f_{k}(\psi, \varphi)$ : 


$$
\begin{gathered}
f_{1}(\psi)=\frac{1}{\psi}, \\
f_{2}(\psi)=\frac{1}{\psi^{3}}, \\
f_{3}(\psi)=3^{3} \frac{1}{\psi^{5}}, \\
f_{4}(\psi)=5^{2} \frac{1}{\psi^{7}}, \\
\vdots .
\end{gathered}
$$

Putting the values of $f_{n}(\psi)(n \geq 1)$ in (44), we get

$$
\begin{gathered}
U(\psi, s)=s^{2}(\psi)+s^{\alpha+2} f_{1}(\psi)+s^{2 \alpha+2} f_{2}(\psi)+s^{3 \alpha+2} f_{3}(\psi)+\cdots \\
U(\psi, s)=s^{2}(\psi)+\frac{s^{\alpha+2}}{\psi}+\frac{s^{2 \alpha+2}}{\psi^{3}}+\frac{3^{2} s^{3 \alpha+2}}{\psi^{5}}+\cdots
\end{gathered}
$$

Using inverse Elzaki transform to (48), we get

$u(\psi, \tau)=\psi+\frac{1}{\psi} \frac{\tau^{\alpha}}{\Gamma(\alpha+2)}+\frac{1}{\psi^{3}} \frac{\tau^{2 \alpha}}{\Gamma(2 \alpha+2)}+\frac{3^{2}}{\psi^{5}} \frac{\tau^{3 \alpha}}{\Gamma(3 \alpha+2)}+\cdots$

Putting $\alpha=1$, we have

$$
\begin{aligned}
& u(\psi, \tau)=\psi+\frac{1}{\psi} t+\frac{1}{\psi^{3}} \frac{\tau^{2}}{2 !}+\frac{3^{2}}{\psi^{5}} \frac{\tau^{3}}{3 !}+\cdots \\
& u(\psi, \tau)=\psi+\sum_{n=1}^{\infty} \frac{1^{2} \times 3^{2} \times 5^{3} \times \cdots \times(2 n-3)^{2}}{r^{2 n-1}} \frac{\tau^{n}}{n !}
\end{aligned}
$$

In Figure 3, the RPSTM and the exact results of Example 2 at $\alpha=1$ are shown by graphs, respectively. From the given figures, it can be seen that both the exact and the EDM results are in close contact with each other. Also, in the Figure 4 subgraph, the RPSTM results of Example 2 are calculated at different fractional-order $\alpha=0.8$ and 0.6 . It is investigated that fractional-order problem results are convergent to an integer-order effect as fractional-order analysis to integerorder. The same phenomenon of convergence of fractionalorder solutions towards integral-order solutions is observed.

Example 3. Consider the fractional-order two-dimensional NS equation of the form

$$
\begin{aligned}
& D_{\tau}^{\alpha} u=\rho_{0}\left(\frac{\partial^{2}}{\partial \psi^{2}} u+\frac{\partial^{2}}{\partial \varphi^{2}} u\right)-u \frac{\partial}{\partial \psi} u-v \frac{\partial}{\partial \varphi} u+g \\
& D_{\tau}^{\alpha} v=\rho_{0}\left(\frac{\partial^{2}}{\partial \psi^{2}} v+\frac{\partial^{2}}{\partial \varphi^{2}} v\right)-u \frac{\partial}{\partial \psi} v-v \frac{\partial}{\partial \varphi} v-g
\end{aligned}
$$

$$
\begin{aligned}
& u(\psi, \varphi, 0)=-\sin (\psi+\varphi), \\
& v(\psi, \varphi, 0)=\sin (\psi+\varphi) .
\end{aligned}
$$

Applying Elzaki transform to (51) and using (52), we get

$$
\begin{aligned}
U(\psi, \varphi, s)= & s^{2}(-\sin (\psi+\varphi))+\rho_{0} s^{\alpha} \mathscr{C}_{\tau} \\
& \cdot\left[\mathscr{E}_{\tau}^{-1}\left\{\frac{\partial^{2}}{\partial \psi^{2}} U_{k}(\psi, \varphi, s)+\frac{\partial^{2}}{\partial \varphi^{2}} U_{k}(\psi, \varphi, s)\right\}\right] \\
& -s^{\alpha} \mathscr{E}_{\tau}\left[\mathscr{E}_{\tau}^{-1}\left\{U(\psi, \varphi, s) \frac{\partial}{\partial \psi} U(\psi, \varphi, s)\right\}\right] \\
& -s^{\alpha} \mathscr{E}_{\tau}\left[\mathscr{E}_{\tau}^{-1}\left\{v(\psi, \varphi, s) \frac{\partial}{\partial \varphi} U(\psi, \varphi, s)\right\}\right]+s^{\alpha} \mathscr{E}_{\tau}[g],
\end{aligned}
$$

$$
\begin{aligned}
V(\psi, \varphi, s)= & s^{2}(\sin (\psi+\varphi))+\rho_{0} s^{\alpha} \mathscr{E}_{\tau} \\
& \cdot\left[\mathscr{E}_{\tau}^{-1}\left(\frac{\partial^{2}}{\partial \psi^{2}} V(\psi, \varphi, s)+\frac{\partial^{2}}{\partial \varphi^{2}} V(\psi, \varphi, s)\right)\right] \\
& -s^{\alpha} \mathscr{E}_{\tau}\left[\mathscr{E}_{\tau}^{-1}\left\{U(\psi, \varphi, s) \frac{\partial}{\partial \psi} V(\psi, \varphi, s)\right\}\right] \\
& -s^{\alpha} \mathscr{E}_{\tau}\left[\mathscr{E}_{\tau}^{-1}\left(V(\psi, \varphi, s) \frac{\partial}{\partial \varphi} V(\psi, \varphi, s)\right)\right]-s^{\alpha} \mathscr{E}_{\tau}[g] .
\end{aligned}
$$

The $k$ th truncated term series of (53) is

$$
\begin{aligned}
& U_{k}(\psi, \varphi, s)=-\sin (\psi+\varphi) s^{2}+\sum_{n=1}^{k} s^{n \alpha+2} f_{n}(\psi, \varphi), \\
& V_{k}(\psi, \varphi, s)=\sin (\psi+\varphi) s^{2}+\sum_{n=1}^{k} s^{n \alpha+2} g_{n}(\psi, \varphi),
\end{aligned}
$$

and the $k$ th Elzaki residual function is

$$
\begin{aligned}
\mathscr{E}_{\tau} \operatorname{Res}_{k}(\psi, \varphi, s)= & U_{k}(\psi, \varphi, s)-(-\sin (\psi+\varphi)) s^{2}-\rho_{0} s^{\alpha} \mathscr{E}_{\tau} \\
& \cdot\left[\mathscr{E}_{\tau}^{-1}\left\{\frac{\partial^{2}}{\partial \psi^{2}} U_{k}(\psi, \varphi, s)+\frac{\partial^{2}}{\partial \varphi^{2}} U_{k}(\psi, \varphi, s)\right\}\right] \\
& +s^{\alpha} \mathscr{E}_{\tau}\left[\mathscr{E}_{\tau}^{-1}\left\{U_{k}(\psi, \varphi, s) \frac{\partial}{\partial \psi} U_{k}(\psi, \varphi, s)\right\}\right] \\
& +s^{\alpha} \mathscr{E}_{\tau}\left[\mathscr{E}_{\tau}^{-1}\left\{V_{k}(\psi, \varphi, s) \frac{\partial}{\partial \varphi} U_{k}(\psi, \varphi, s)\right\}\right] \\
& -g s^{\alpha+2}, \\
\mathscr{E}_{\tau} \operatorname{Re~s}_{k}(\psi, \varphi, s)= & \left.\left.V_{k}(\psi, \varphi, s)-\sin (\psi+\varphi) s^{2}-\rho_{0} s^{\alpha} \mathscr{E}_{\tau}\right)\right\} \\
& +\left[\mathscr{E}_{\tau}^{-1}\left\{\frac{\partial^{2}}{\partial \psi^{2}} V_{k}(\psi, \varphi, s)+\frac{\partial^{2}}{\partial \varphi^{2}} V_{k}(\psi, \varphi, s)\right\}\right] \\
& +s^{\alpha} \mathscr{E}_{\tau}\left[\mathscr{E}_{\tau}^{-1}\left\{U_{k}(\psi, \varphi, s) \frac{\partial}{\partial \psi} V_{k}(\psi, \varphi, s)\right\}\right] \\
& +s^{\alpha} \mathscr{E}_{\tau}\left[\mathscr{E}_{\tau}^{-1}\left\{V_{k}(\psi, \varphi, s) \frac{\partial}{\partial \varphi} V_{k}(\psi, \varphi, s)\right\}\right] \\
& +g s^{\alpha+2} .
\end{aligned}
$$

with initial condition 

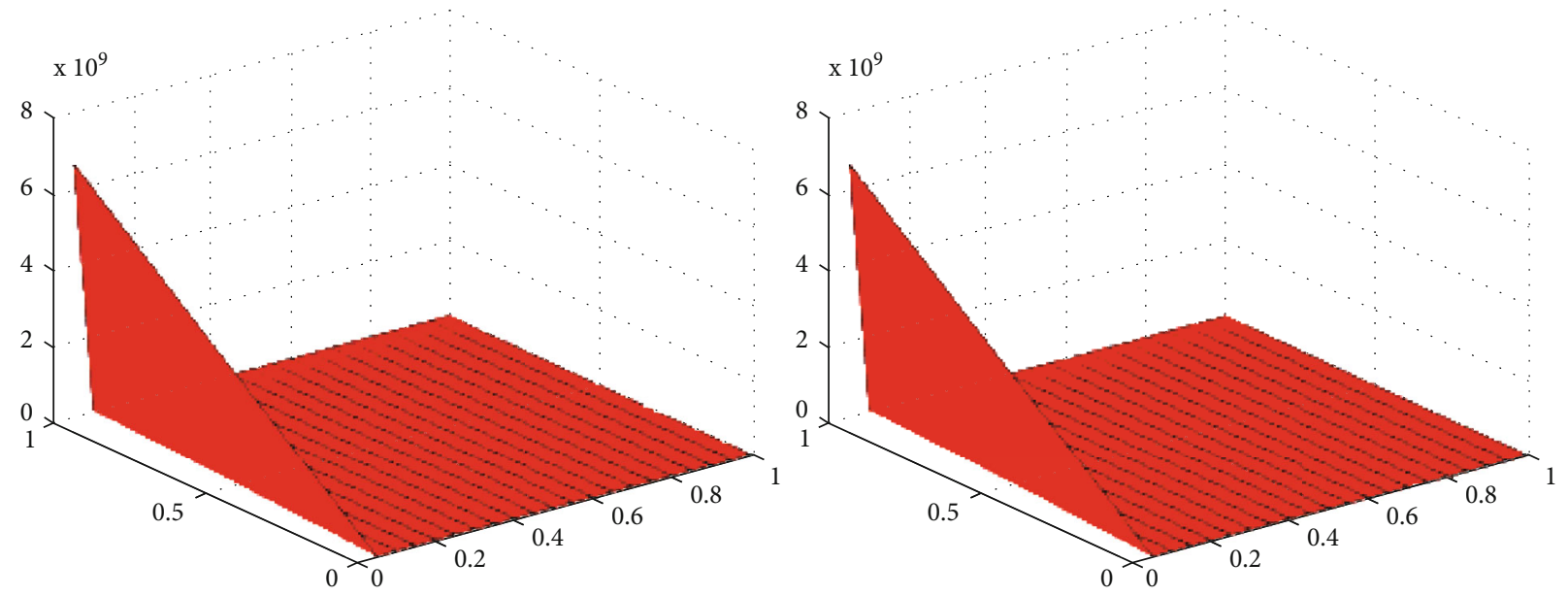

Figure 3: Graph of exact and analytical results of Problem 2.
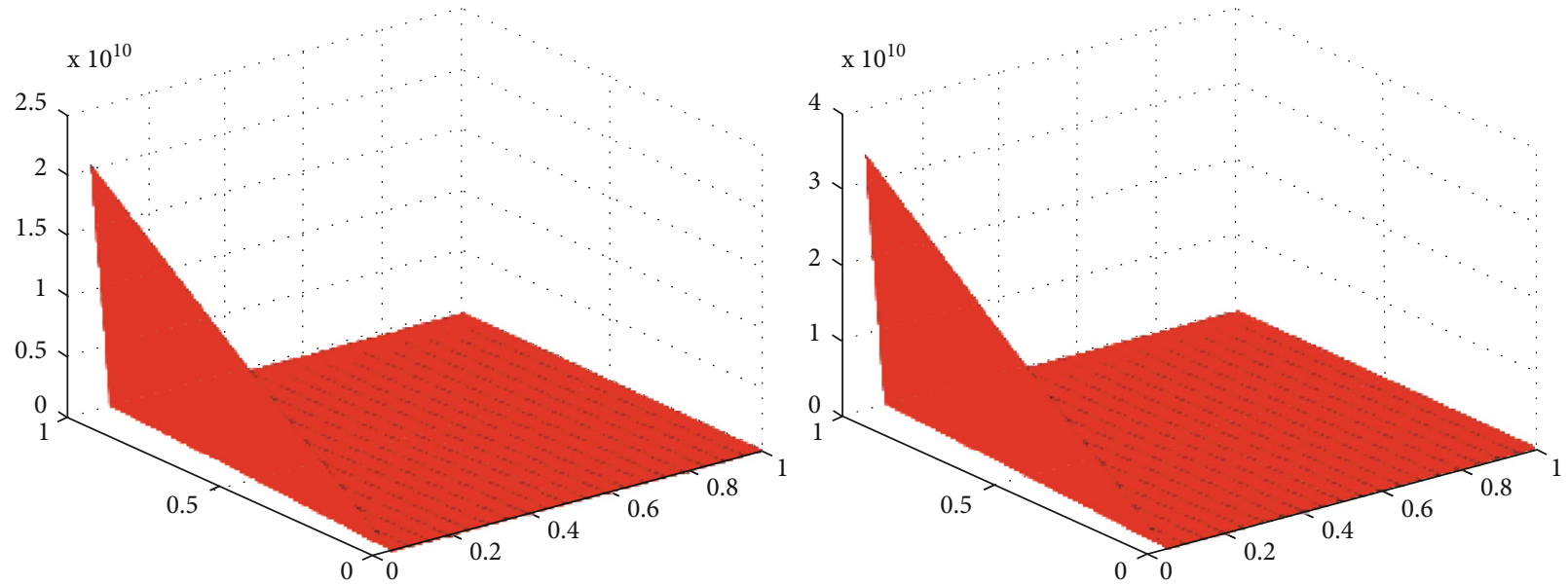

Figure 4: The fractional order of $\alpha=0.8$ and 0.6 of Problem 2.
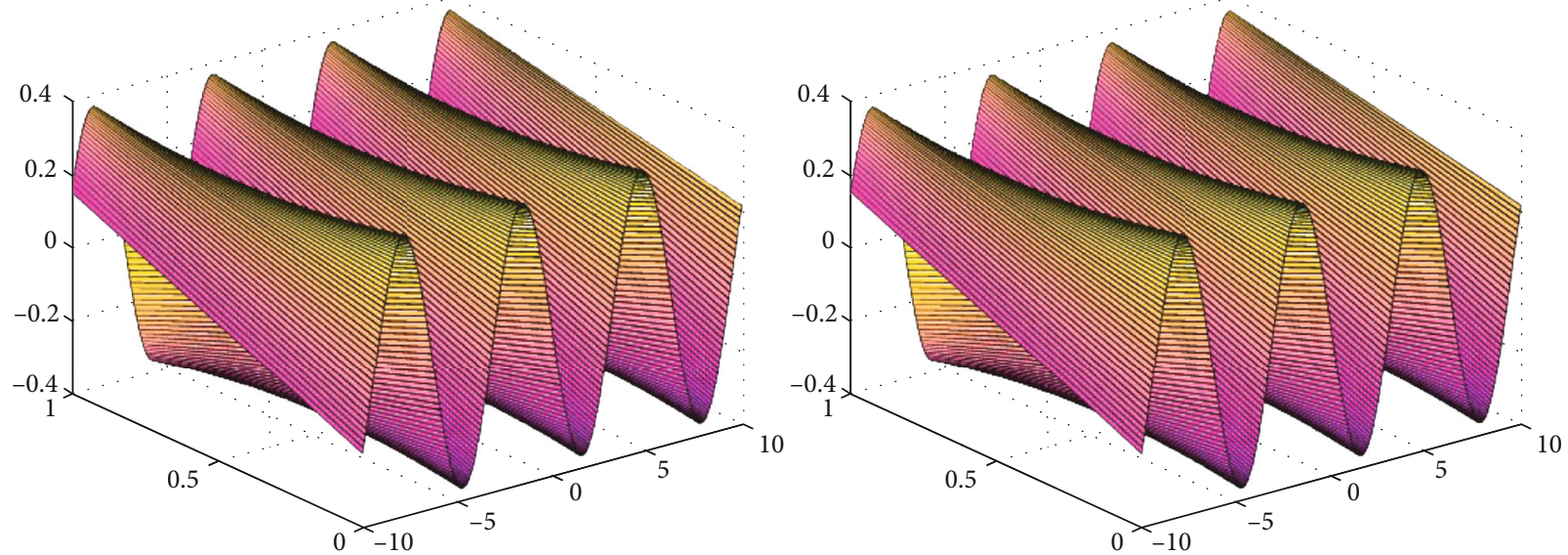

FIgURE 5: Graph of exact and analytical results of Problem 3.

Now, to determine $f_{k}(\psi, \varphi)$ and $g_{k}(\psi, \varphi), k=1,2,3, \cdots$, we substitute the $k$ th truncated series (54) into the $k$ th Elzaki residual function (55), multiply the resulting equation by $s^{k \alpha+2}$
, and then solve recursively the relation $\lim _{s \rightarrow \infty}\left[s^{k \alpha+2} \operatorname{Re} s_{k}(\psi\right.$ $, \varphi, s)]=0, k=1,2,3, \cdots$, for $f_{k}$ and $g_{k}$. The following are the first several components of the series $f_{k}(\psi, \varphi)$ and $g_{k}(\psi, \varphi)$ : 


$$
\begin{aligned}
& f_{1}(\psi, \varphi)=2 \rho_{0} \sin (\psi+\varphi)+g, \\
& g_{1}(\psi, \varphi)=-2 \rho_{0} \sin (\psi+\varphi)-g, \\
& f_{2}(\psi, \varphi)=-\left(2 \rho_{0}\right)^{2} \sin (\psi+\varphi), \\
& g_{2}(\psi, \varphi)=\left(2 \rho_{0}\right)^{2} \sin (\psi+\varphi), \\
& f_{3}(\psi, \varphi)=\left(2 \rho_{0}\right)^{3} \sin (\psi+\varphi), \\
& g_{3}(\psi, \varphi)=-\left(2 \rho_{0}\right)^{3} \sin (\psi+\varphi),
\end{aligned}
$$

Putting the values of $f_{n}(\psi, \varphi)$ and $g_{n}(\psi, \varphi)(n \geq 1)$ in (54), we have

$$
\begin{aligned}
U(\psi, \varphi, s)=- & \sin (\psi+\varphi) s^{2}+f_{1}(\psi, \varphi) s^{\alpha+2}+f_{2}(\psi, \varphi) s^{2 \alpha+2} \\
& +f_{3}(\psi, \varphi) s^{3 \alpha+2}+\cdots, \\
V(\psi, \varphi, s)= & \sin (\psi+\varphi) s^{2}+g_{1}(\psi, \varphi) s^{\alpha+2}+g_{2}(\psi, \varphi) s^{2 \alpha+2} \\
& +g_{3}(\psi, \varphi) s^{3 \alpha+2}+\cdots, \\
U(\psi, \varphi, s)=- & \sin (\psi+\varphi) s^{2}+\left(2 \rho_{0} \sin (\psi+\varphi)+g\right) s^{\alpha+2} \\
& -\left(\left(2 \rho_{0}\right)^{2} \sin (\psi+\varphi)\right) s^{2 \alpha+2} \\
& +\left(\left(2 \rho_{0}\right)^{3} \sin (\psi+\varphi)\right) s^{3 \alpha+2}+\cdots, \\
V(\psi, \varphi, s)= & \sin (\psi+\varphi) s^{2}-\left(2 \rho_{0} \sin (\psi+\varphi)-g\right) s^{\alpha+2} \\
& \left.+\left(\left(2 \rho_{0}\right)^{2} \sin (\psi+\varphi)\right)\right) s^{2 \alpha+2} \\
& \left.-\left(\left(2 \rho_{0}\right)^{3} \sin (\psi+\varphi)\right)\right) s^{3 \alpha+2}+\cdots, \\
& \left.-\left(2 \rho_{0}\right)^{3} s^{3 \alpha+2}+\cdots\right]-g s^{\alpha+2} . \\
U(\psi, \varphi, s)=- & \sin (\psi+\varphi)\left[s^{2}-2 \rho_{0} s^{\alpha+2}+\left(2 \rho_{0}\right)^{2} s^{2 \alpha+2}\right. \\
& \left.-\left(2 \rho_{0}\right)^{3} s^{3 \alpha+2}+\cdots\right]+g s^{\alpha+2}, \\
V(\psi, \varphi, s)= & \sin (\psi+\varphi)\left[s^{2}-2 \rho_{0} s^{\alpha+2}+\left(2 \rho_{0}\right)^{2} s^{2 \alpha+2}\right. \\
& (57) \\
&
\end{aligned}
$$

Using inverse Elzaki transform, we get

$$
\begin{aligned}
u(\psi, \varphi, \tau)= & -\sin (\psi+\varphi)\left[1-\frac{2 \rho_{0} \tau^{\alpha}}{\Gamma(\alpha+2)}+\frac{\left(2 \rho_{0}\right)^{2} \tau^{2 \alpha}}{\Gamma(2 \alpha+2)}-\frac{\left(2 \rho_{0}\right)^{3} \tau^{3 \alpha}}{\Gamma(3 \alpha+2)}+\cdots\right] \\
& +g \frac{\tau^{\alpha}}{\Gamma(\alpha+2)}, \\
v(\psi, \varphi, \tau)= & \sin (\psi+\varphi)\left[1-\frac{2 \rho_{0} \tau^{\alpha}}{\Gamma(\alpha+2)}+\frac{\left(2 \rho_{0}\right)^{2} \tau^{2 \alpha}}{\Gamma(2 \alpha+2)}-\frac{\left(2 \rho_{0}\right)^{3} \tau^{3 \alpha}}{\Gamma(3 \alpha+2)}+\cdots\right] \\
& -g \frac{\tau^{\alpha}}{\Gamma(\alpha+2)} .
\end{aligned}
$$

Putting $\alpha=1$, we get the solution in closed form

$$
\begin{aligned}
& u(\psi, \varphi, \tau)=-\sin (\psi+\varphi) e^{-2 \rho_{0} \tau}+g, \\
& v(\psi, \varphi, \tau)=\sin (\psi+\varphi) e^{-2 \rho_{0} \tau}-g .
\end{aligned}
$$

In Figures 5 and 6 , the RPSTM and the exact results of Example 3 at $\alpha=1$ are shown by graphs, respectively. From the given figures, it can be seen that both the exact and the RPSTM results are in close contact with each other. Also, in the Figure 7 and 8 subgraph, the RPSTM results of Example 3 are calculated at different fractional-order $\alpha=0.8$ and 0.6 . It is investigated that fractional-order problem results are convergent to an integer-order effect as fractional-order analysis to integer-order. The same phenomenon of convergence of fractional-order solutions towards integral-order solutions is observed.

Example 4. Consider the fractional-order two-dimensional NS equation as

$$
\begin{aligned}
& D_{\tau}^{\alpha} u=\rho_{0}\left(\frac{\partial^{2}}{\partial \psi^{2}} u+\frac{\partial^{2}}{\partial \varphi^{2}} u\right)-u \frac{\partial}{\partial \psi} u-v \frac{\partial}{\partial \varphi} u+g \\
& D_{\tau}^{\alpha} v=\rho_{0}\left(\frac{\partial^{2}}{\partial \psi^{2}} v+\frac{\partial^{2}}{\partial \varphi^{2}} v\right)-u \frac{\partial}{\partial \psi} v-v \frac{\partial}{\partial \varphi} v-g
\end{aligned}
$$

with initial condition

$$
\begin{gathered}
u(\psi, \varphi, 0)=-e^{\psi+\varphi}, \\
v(\psi, \varphi, 0)=e^{\psi+\varphi} .
\end{gathered}
$$

Applying Elzaki transform to (60) and using (61), we get

$$
\begin{aligned}
U(\psi, \varphi, s)= & -e^{\psi+\varphi} s^{2}+\rho_{0} s^{\alpha} \mathscr{E}_{\tau} \\
& \cdot\left[\mathscr{E}_{\tau}^{-1}\left\{\frac{\partial^{2}}{\partial \psi^{2}} U_{k}(\psi, \varphi, s)+\partial^{2} \partial \varphi^{2} U_{k}(\psi, \varphi, s)\right\}\right] \\
& -s^{\alpha} \mathscr{E}_{\tau}\left[\mathscr{E}_{\tau}^{-1}\left\{U(\psi, \varphi, s) \frac{\partial}{\partial \psi} U(\psi, \varphi, s)\right\}\right] \\
& -s^{\alpha} \mathscr{E}_{\tau}\left[\mathscr{E}_{\tau}^{-1}\left\{v(\psi, \varphi, s) \frac{\partial}{\partial \varphi} U(\psi, \varphi, s)\right\}\right] \\
& +s^{\alpha} \mathscr{E}_{\tau}[g], \\
V(\psi, \varphi, s)= & e^{\psi+\varphi^{2}} s^{2}+\rho_{0} s^{\alpha} \mathscr{E}_{\tau} \\
& \cdot\left[\mathscr{E}_{\tau}^{-1}\left\{\frac{\partial^{2}}{\partial \psi^{2}} V(\psi, \varphi, s)+\frac{\partial^{2}}{\partial \varphi^{2}} V(\psi, \varphi, s)\right\}\right] \\
& -s^{\alpha} \mathscr{E}_{\tau}\left[\mathscr{E}_{\tau}^{-1}\left\{U(\psi, \varphi, s) \frac{\partial}{\partial \psi} V(\psi, \varphi, s)\right\}\right] \\
& -s^{\alpha} \mathscr{E}_{\tau}\left[\mathscr{E}_{\tau}^{-1}\left\{V(\psi, \varphi, s) \frac{\partial}{\partial \varphi} V(\psi, \varphi, s)\right\}\right] \\
& -s^{\alpha} \mathscr{E}_{\tau}[g] .
\end{aligned}
$$





Figure 6: Graph of exact and analytical results of Problem 3.
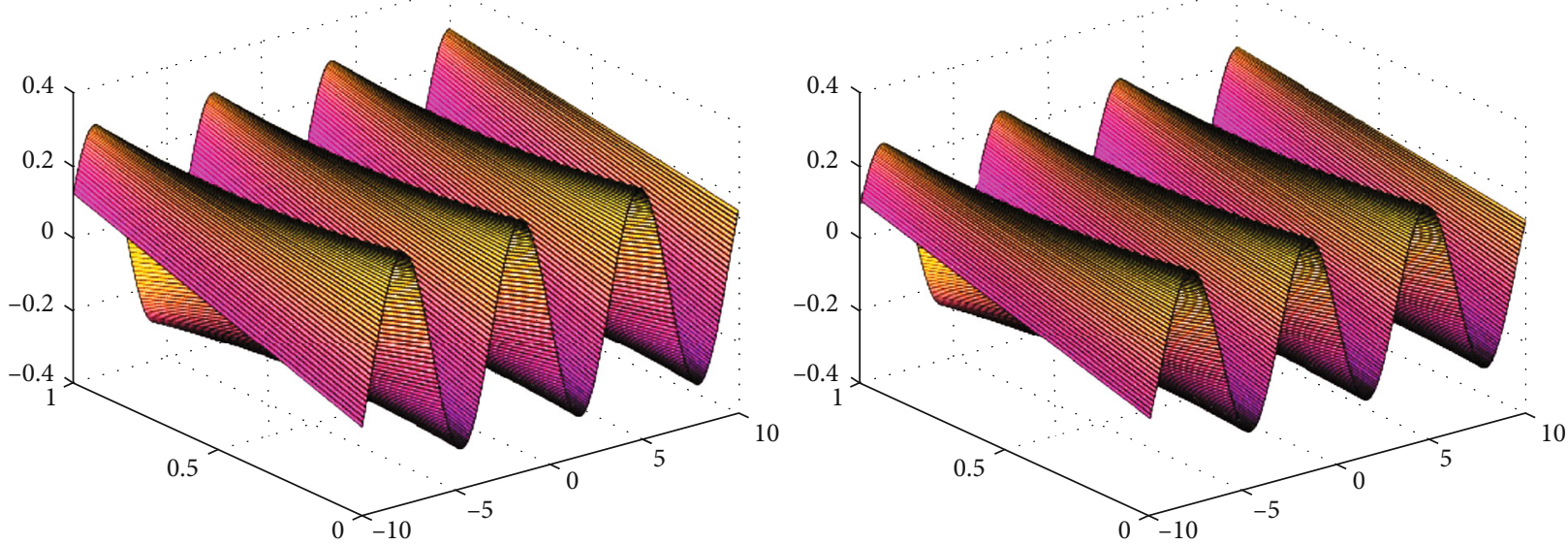

Figure 7: The fractional order of $\alpha=0.8$ and 0.6 of Problem 3 .
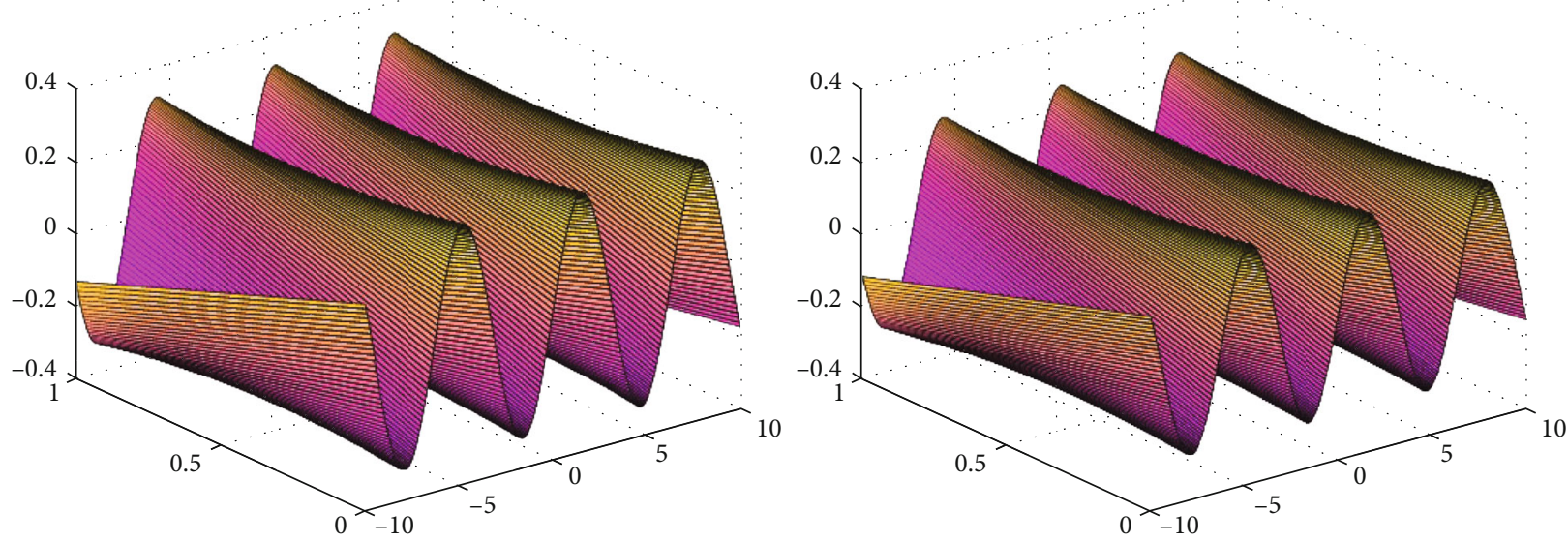

Figure 8: The fractional order of $\alpha=0.8$ and 0.6 of Problem 3 . 

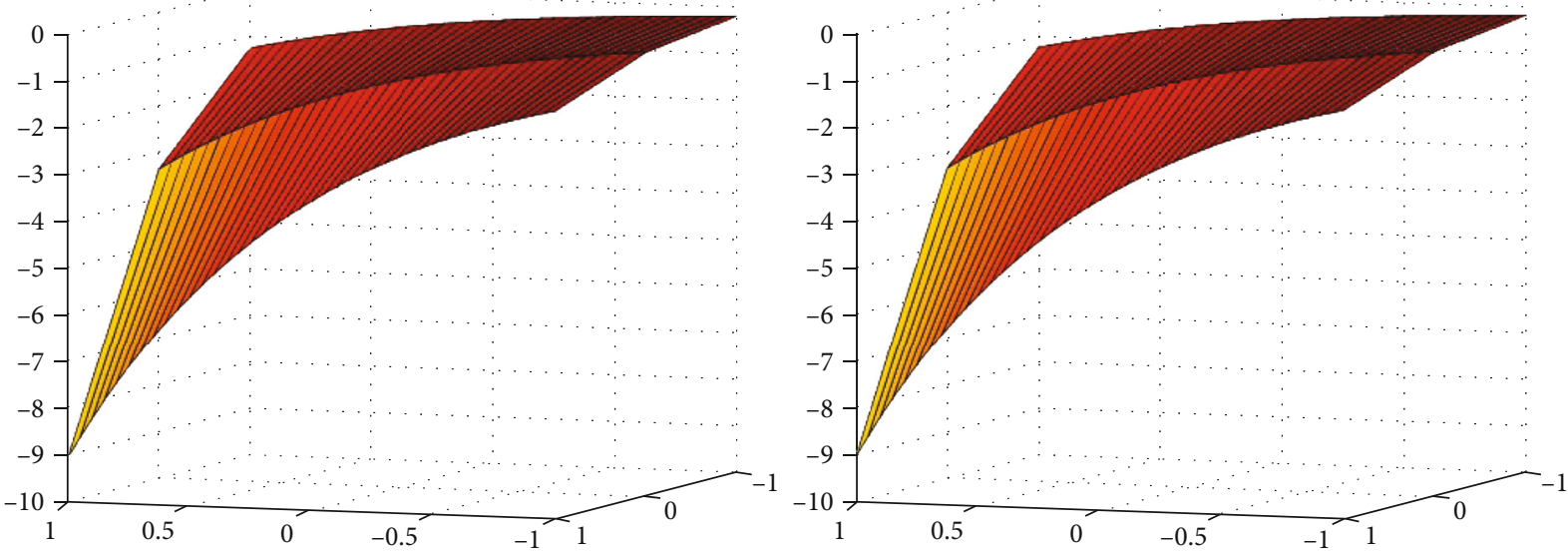

FIgURE 9: Graph of exact and analytical results of Problem 4.
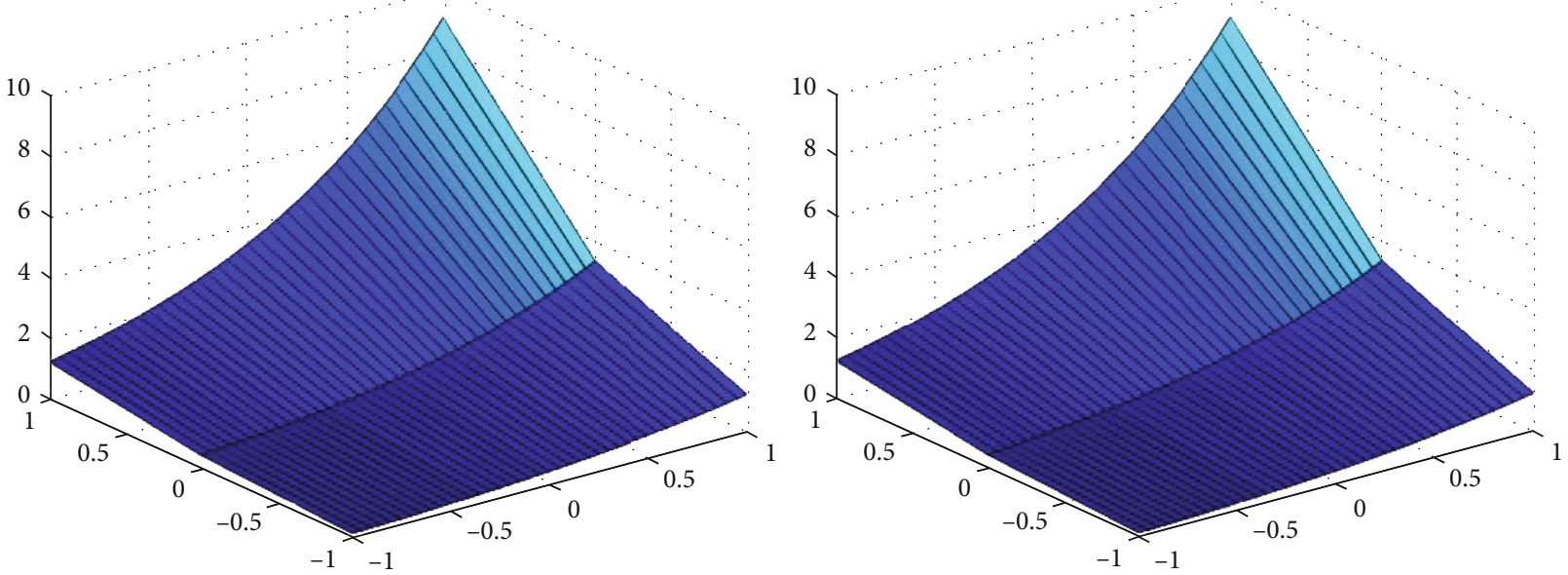

Figure 10: Graph of exact and analytical results of Problem 4.

The $k$ th truncated term series of (62) is

$$
\begin{aligned}
& U_{k}(\psi, \varphi, s)=-e^{\psi+\varphi} s^{2}+\sum_{n=1}^{k} s^{n \alpha+2} f_{n}(\psi, \varphi), \\
& V_{k}(\psi, \varphi, s)=e^{\psi+\varphi} s^{2}+\sum_{n=1}^{k} s^{n \alpha+2} g_{n}(\psi, \varphi),
\end{aligned}
$$

and the $k$ th Elzaki residual function is

$$
\begin{aligned}
\mathscr{E}_{\tau} \operatorname{Re}_{k}(\psi, \varphi, s)= & U_{k}(\psi, \varphi, s)--e^{\psi+\varphi} s^{2}-\rho_{0} s^{\alpha} \mathscr{C}_{\tau} \\
& \cdot\left[\mathscr{E}_{\tau}^{-1}\left\{\frac{\partial^{2}}{\partial \psi^{2}} U_{k}(\psi, \varphi, s)+\frac{\partial^{2}}{\partial \varphi^{2}} U_{k}(\psi, \varphi, s)\right\}\right] \\
& +s^{\alpha} \mathscr{E}_{\tau}\left[\mathscr{E}_{\tau}^{-1}\left\{U_{k}(\psi, \varphi, s) \frac{\partial}{\partial \psi} U_{k}(\psi, \varphi, s)\right\}\right] \\
& +s^{\alpha} \mathscr{E}_{\tau}\left[\mathscr{E}_{\tau}^{-1}\left\{V_{k}(\psi, \varphi, s) \frac{\partial}{\partial \varphi} U_{k}(\psi, \varphi, s)\right\}\right] \\
& -g \frac{1}{s^{\alpha+2 p}},
\end{aligned}
$$

$$
\begin{aligned}
\mathscr{E}_{\tau} \operatorname{Re} s_{k}(\psi, \varphi, s)= & V_{k}(\psi, \varphi, s)-e^{\psi+\varphi} s^{2}-\rho_{0} s^{\alpha} \mathscr{E}_{\tau} \\
& \cdot\left[\mathscr{E}_{\tau}^{-1}\left\{\frac{\partial^{2}}{\partial \psi^{2}} V_{k}(\psi, \varphi, s)+\frac{\partial^{2}}{\partial \varphi^{2}} V_{k}(\psi, \varphi, s)\right\}\right] \\
& +s^{\alpha} \mathscr{E}_{\tau}\left[\mathscr{E}_{\tau}^{-1}\left\{U_{k}(\psi, \varphi, s) \frac{\partial}{\partial \psi} V_{k}(\psi, \varphi, s)\right\}\right] \\
& +s^{\alpha} \mathscr{E}_{\tau}\left[\mathscr{E}_{\tau}^{-1}\left\{V_{k}(\psi, \varphi, s) \frac{\partial}{\partial \varphi} V_{k}(\psi, \varphi, s)\right\}\right] \\
& +g \frac{1}{s^{\alpha+2}} .
\end{aligned}
$$

Now, to determine $f_{k}(\psi, \varphi)$ and $g_{k}(\psi, \varphi), k=1,2,3, \cdots$, we substitute the $k$ th truncated series (63) into the $k$ th Elzaki residual function (64), multiply the resulting equation by $s^{k \alpha+2}$, and then solve recursively the relation $\lim _{s \rightarrow \infty}\left[s^{k \alpha+2}\right.$ Re $\left.s_{k}(\psi, \varphi, s)\right]=0, k=1,2,3, \cdots$, for $f_{k}$ and $g_{k}$. The following are the first several components of the series $f_{k}(\psi, \varphi)$ and $g_{k}(\psi, \varphi)$ : 

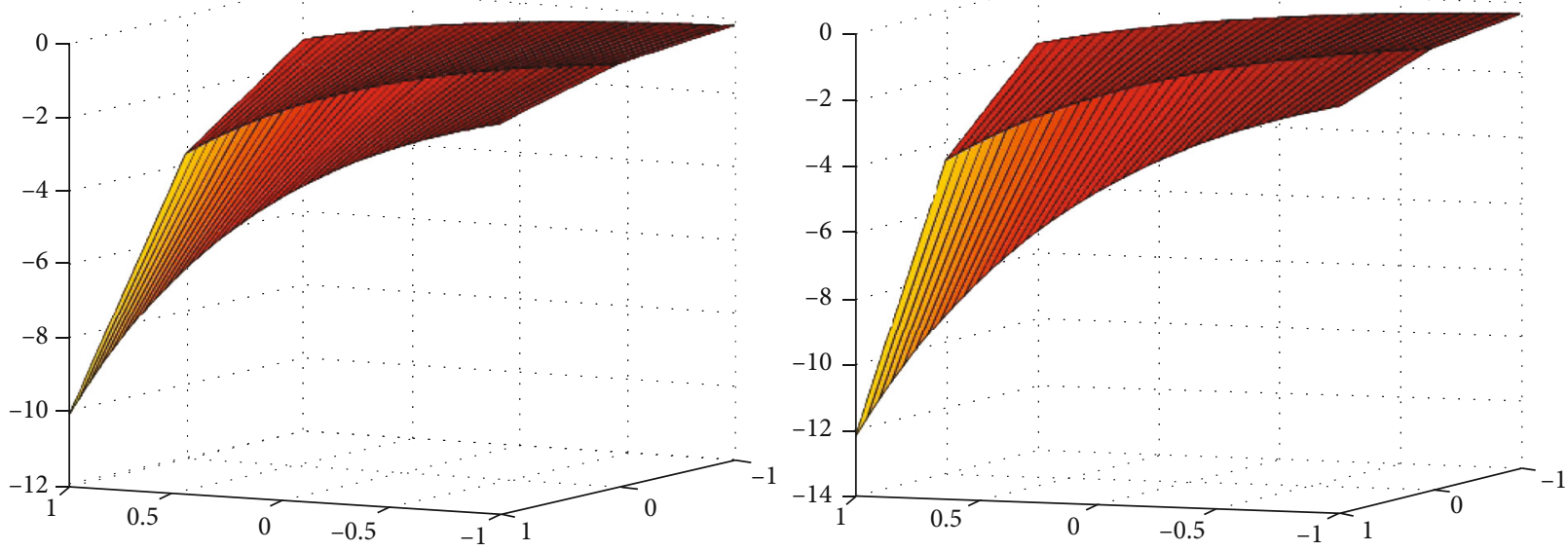

Figure 11: The fractional order of $\alpha=0.8$ and 0.6 of Problem 4.
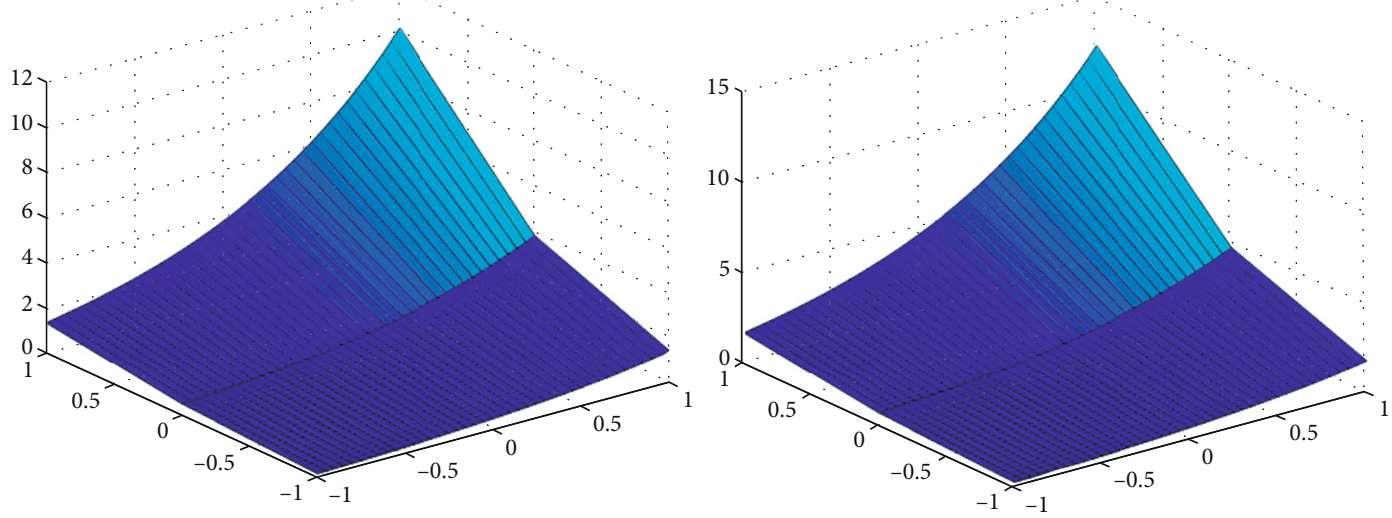

Figure 12: The fractional order of $\alpha=0.8$ and 0.6 of Problem 4.

$$
\begin{aligned}
& f_{1}(\psi, \varphi)=-2 \rho_{0} e^{\psi+\varphi}+g, \\
& g_{1}(\psi, \varphi)=2 \rho_{0} e^{\psi+\varphi}-g, \\
& f_{2}(\psi, \varphi)=-\left(2 \rho_{0}\right)^{2} e^{\psi+\varphi}, \\
& g_{2}(\psi, \varphi)=\left(2 \rho_{0}\right)^{2} e^{\psi+\varphi}, \\
& f_{3}(\psi, \varphi)=-\left(2 \rho_{0}\right)^{3} e^{\psi+\varphi}, \\
& g_{3}(\psi, \varphi)=\left(2 \rho_{0}\right)^{3} e^{\psi+\varphi},
\end{aligned}
$$

Putting the values of $f_{n}(\psi, \varphi)$ and $g_{n}(\psi, \varphi)(n \geq 1)$ in (63), we have

$$
\begin{aligned}
U(\psi, \varphi, s)= & -e^{\psi+\varphi} s^{2}+f_{1}(\psi, \varphi) s^{\alpha+2}+f_{2}(\psi, \varphi) s^{2 \alpha+2} \\
& +f_{3}(\psi, \varphi) s^{3 \alpha+2}+\cdots, \\
V(\psi, \varphi, s)= & e^{\psi+\varphi} s^{2}+g_{1}(\psi, \varphi) s^{\alpha+2}+g_{2}(\psi, \varphi) s^{2 \alpha+2} \\
& +g_{3}(\psi, \varphi) s^{3 \alpha+2}+\cdots,
\end{aligned}
$$

$$
\begin{aligned}
& U(\psi, \varphi, s)=-e^{\psi+\varphi} s^{2}-2 \rho_{0} e^{\psi+\varphi}+g s^{\alpha+2}-\left(2 \rho_{0}\right)^{2} e^{\psi+\varphi} s^{2 \alpha+2} \\
&-\left(2 \rho_{0}\right)^{3} e^{\psi+\varphi} s^{3 \alpha+2}+\cdots, \\
& V(\psi, \varphi, s)= e^{\psi+\varphi} s^{2}+2 \rho_{0} e^{\psi+\varphi}-g s^{\alpha+2}+\left(2 \rho_{0}\right)^{2} e^{\psi+\varphi} s^{2 \alpha+2} \\
&+\left(2 \rho_{0}\right)^{3} e^{\psi+\varphi} s^{3 \alpha+2}+\cdots, \\
& U(\psi, \varphi, s)=-e^{\psi+\varphi}\left[s^{2}+2 \rho_{0} s^{\alpha+2}+\left(2 \rho_{0}\right)^{2} s^{2 \alpha+2}+\left(2 \rho_{0}\right)^{3} s^{3 \alpha+2}+\cdots\right]+g s^{\alpha+2},
\end{aligned}
$$

$V(\psi, \varphi, s)=e^{\psi+\varphi}\left[s^{2}+2 \rho_{0} s^{\alpha+2}+\left(2 \rho_{0}\right)^{2} s^{2 \alpha+2}+\left(2 \rho_{0}\right)^{3} s^{3 \alpha+2}+\cdots\right]-g s^{\alpha+2}$

Using inverse Elzaki transform, we get

$$
\begin{aligned}
u(\psi, \varphi, \tau)= & -e^{\psi+\varphi}\left[1+\frac{2 \rho_{0} \tau^{\alpha}}{\Gamma(\alpha+2)}+\frac{\left(2 \rho_{0}\right)^{2} \tau^{2 \alpha}}{\Gamma(2 \alpha+2)}+\frac{\left(2 \rho_{0}\right)^{3} \tau^{3 \alpha}}{\Gamma(3 \alpha+2)}+\cdots\right] \\
& +g \frac{\tau^{\alpha}}{\Gamma(\alpha+2)}
\end{aligned}
$$




$$
\begin{aligned}
v(\psi, \varphi, \tau)= & e^{\psi+\varphi}\left[1+\frac{2 \rho_{0} \tau^{\alpha}}{\Gamma(\alpha+2)}+\frac{\left(2 \rho_{0}\right)^{2} \tau^{2 \alpha}}{\Gamma(2 \alpha+2)}+\frac{\left(2 \rho_{0}\right)^{3} \tau^{3 \alpha}}{\Gamma(3 \alpha+2)}+\cdots\right] \\
& -g \frac{\tau^{\alpha}}{\Gamma(\alpha+2)} .
\end{aligned}
$$

Putting $\alpha=1$, we get the solution in closed form

$$
\begin{aligned}
& u(\psi, \varphi, \tau)=-e^{\psi+\varphi+2 \rho_{0} \tau}+g, \\
& v(\psi, \varphi, \tau)=e^{\psi+\varphi+2 \rho_{0} \tau}-g .
\end{aligned}
$$

In Figures 9 and 10, the RPSTM and the exact results of Example 4 at $\alpha=1$ are shown by graphs, respectively. From the given figures, it can be seen that both the exact and the RPSTM results are in close contact with each other. Also, in the Figure 11 and 12 subgraph, the RPSTM results of Example 4 are calculated at different fractional-order $\alpha=0.8$ and 0.6 . It is investigated that fractional-order problem results are convergent to an integer-order effect as fractional-order analysis to integer-order. The same phenomenon of convergence of fractional-order solutions towards integral-order solutions is observed.

\section{Conclusions}

In this article, a modified method constructed by a mixture of the residual power series and Elzaki transformation operator is presented to solve fractional-order Navier-Stokes models. The merit of the modified technique is to reduce the size of computational work needed to find the result in a power series form whose coefficient to be calculated is in successive algebraic steps. The technique gives a series form of results that converges very fast in physical models. It is predicted that this article achieved results which will be useful for further analysis of the complicated nonlinear physical problems. The calculations of this technique are very straightforward and simple. Thus, we deduce that this technique can be implemented to solve several schemes of nonlinear fractional-order partial differential equations.

\section{Data Availability}

The numerical data used to support the findings of this study are included within the article.

\section{Conflicts of Interest}

The authors declare that there are no conflicts of interest regarding the publication of this article.

\section{Acknowledgments}

This work was supported by the Korea Institute of Energy Technology Evaluation and Planning (KETEP) grant funded by the Korea government (MOTIE) (20202020900060, The Development and Application of Operational Technology in Smart Farm Utilizing Waste Heat from Particulates Reduced Smokestack).

\section{References}

[1] A. Prakash, P. Veeresha, D. G. Prakasha, and M. Goyal, "A new efficient technique for solving fractional coupled NavierStokes equations using $q$-homotopy analysis transform method," Pramana, vol. 93, no. 1, pp. 1-10, 2019.

[2] A. Rauf, Y. Mahsud, I. A. Mirza, and Q. Rubbab, "Multi-layer flows of immiscible fractional Maxwell fluids with generalized thermal flux," Chinese Journal of Physics, vol. 62, pp. 313-334, 2019.

[3] A. Shah, N. A. Nehad, T. Elnaqeeb, and M. M. Rashidi, "Magnetohydrodynamic free convection flows with thermal memory over a moving vertical plate in porous medium," Journal of Applied and Computational Mechanics, vol. 5, no. 1, pp. 150-161, 2019.

[4] M. El-Shahed and A. Salem, "On the generalized NavierStokes equations," Applied Mathematics and Computation, vol. 156, no. 1, pp. 287-293, 2004.

[5] S. Momani and Z. Odibat, "Analytical solution of a timefractional Navier-Stokes equation by Adomian decomposition method," Applied Mathematics and Computation, vol. 177, no. 2, pp. 488-494, 2006.

[6] Z. Z. Ganji, D. D. Ganji, A. D. Ganji, and M. Rostamian, "Analytical solution of time-fractional Navier-Stokes equation in polar coordinate by homotopy perturbation method," Numerical Methods for Partial Differential Equations, vol. 26, no. 1, pp. 117-124, 2010.

[7] B. K. Singh and P. Kumar, "FRDTM for numerical simulation of multi-dimensional, time-fractional model of Navier-Stokes equation," Ain Shams Engineering Journal, vol. 9, no. 4, pp. 827-834, 2018.

[8] D. S. Oliveira and E. C. de Oliveira, "Analytical solutions for Navier-Stokes equations with Caputo fractional derivative," SeMA Journal, vol. 78, no. 1, pp. 137-154, 2021.

[9] J. Zhang and J. R. Wang, "Numerical analysis for NavierStokes equations with time fractional derivatives," Applied Mathematics and Computation, vol. 336, pp. 481-489, 2018.

[10] S. S. Ravindran, "Exact boundary controllability of Galerkin approximations of Navier-Stokes system for soret convection," An International Journal of Optimization and Control: Theories \& Applications (IJOCTA), vol. 5, no. 2, pp. 41-49, 2015.

[11] A. Cibik and F. Yilmaz, "Brezzi-Pitkaranta stabilization and a priori error analysis for the Stokes control," An International Journal of Optimization and Control: Theories \& Applications (IJOCTA), vol. 7, no. 1, pp. 75-82, 2017.

[12] D. Kumar, J. Singh, and S. Kumar, "A fractional model of Navier-Stokes equation arising in unsteady flow of a viscous fluid," Journal of the Association of Arab Universities for Basic and Applied Sciences, vol. 17, no. 1, pp. 14-19, 2015.

[13] R. M. Jena and S. Chakraverty, "Solving time-fractional Navier-Stokes equations using homotopy perturbation Elzaki transform," SN Applied Sciences, vol. 1, no. 1, pp. 1-13, 2019.

[14] M. Alquran, M. Ali, M. Alsukhour, and I. Jaradat, "Promoted residual power series technique with Laplace transform to solve some time-fractional problems arising in physics," Results in Physics, vol. 19, p. 103667, 2020.

[15] A. Çibik, "The effect of a sparse grad-div stabilization on control of stationary Navier-Stokes equations," Journal of 
Mathematical Analysis and Applications, vol. 437, no. 1, pp. 613-628, 2016.

[16] O. A. Arqub, A. El-Ajou, A. S. Bataineh, and I. Hashim, "A representation of the exact solution of generalized LaneEmden equations using a new analytical method," Abstract and Applied Analysis, vol. 2013, Article ID 378593, 10 pages, 2013.

[17] A. Arqub, Z. A.-H. Omar, R. Al-Badarneh, and S. Momani, “A reliable analytical method for solving higher-order initial value problems," Discrete Dynamics in Nature and Society, vol. 2013, Article ID 673829, 12 pages, 2013.

[18] A. El-Ajou, O. A. Arqub, Z. Al Zhour, and S. Momani, "New results on fractional power series: theories and applications," Entropy, vol. 15, no. 12, pp. 5305-5323, 2013.

[19] O. A. Arqub, A. El-Ajou, Z. Al Zhour, and S. Momani, "Multiple solutions of nonlinear boundary value problems of fractional order: a new analytic iterative technique," Entropy, vol. 16, no. 1, pp. 471-493, 2014.

[20] A. El-Ajou, O. A. Arqub, and S. Momani, "Approximate analytical solution of the nonlinear fractional KdV-Burgers equation: a new iterative algorithm," Journal of Computational Physics, vol. 293, pp. 81-95, 2015.

[21] O. A. Arqub, A. El-Ajou, and S. Momani, "Constructing and predicting solitary pattern solutions for nonlinear timefractional dispersive partial differential equations," Journal of Computational Physics, vol. 293, pp. 385-399, 2015.

[22] M. Alquran, "Analytical solutions of fractional foam drainage equation by residual power series method," Mathematical Sciences, vol. 8, no. 4, pp. 153-160, 2014.

[23] S.-D. Lin and L. Chia-Hung, "Laplace transform for solving some families of fractional differential equations and its applications," Advances in Difference Equations, vol. 2013, no. 1, p. 9, 2013.

[24] M. Alquran, "Analytical solution of time-fractional twocomponent evolutionary system of order 2 by residual power series method," Journal of Applied Analysis \& Computation, vol. 5, no. 4, pp. 589-599, 2015.

[25] T. M. Elzaki, "The new integral transform Elzaki transform," Global Journal of Pure and Applied Mathematics, vol. 7, no. 1, pp. 57-64, 2011.

[26] T. M. Elzaki, "On the connections between Laplace and Elzaki transforms," Advances in Theoretical and Applied Mathematics, vol. 6, no. 1, pp. 1-11, 2011.

[27] T. M. Elzaki, "On the new integral transform"Elzaki transform"fundamental properties investigations and applications," Global Journal of Mathematical Sciences: Theory and Practical, vol. 4, no. 1, pp. 1-13, 2012. 